\title{
The role of spiral arms in the chemical evolution of galactic disks
}

\author{
Jacques R. D. Lépine ${ }^{1}$, Yuri N. Mishurov ${ }^{2}$, and Irina A. Acharova ${ }^{2}$ \\ ${ }^{1}$ Instituto de Astronomia, Geofísica e Ciências Atmosf., Universidade de São Paulo, 01065-970 \\ São Paulo /SP, Brazil, email: jacques@astro.iag.usp.br \\ ${ }^{2}$ Department of Space Research, Rostov State University, 5, Zorge Street, Rostov-on-Don, \\ 344090, Russia
}

\begin{abstract}
In grand design spiral galaxies, spiral arms play a dominant role in the oxygen enrichment rate, since type II supernovae are the main source of oxygen, and these supernovae only appear in spiral arms. The connection between the spiral structure and the star formation rate explains oxygen abundance gradients that rise outward, in the external regions of a number of galaxies.
\end{abstract}

Keywords. Galaxies: abundances, galaxies: spiral

\section{Introduction}

Models of chemical evolution of the interstellar medium in galactic disks usually ignore the role of the spiral arms. This is surprising, since the two facts, 1)that the star formation rate plays an essential role in the chemical evolution, and 2) that the spiral arms are the dominant mechanism of star formation, are both well accepted. One might think that the exact mechanism that stimulates star formation, in chemical evolution models, does not matter; after all, the models only require a smooth function to describe the rate of star formation as a function of radius, and eventually, a description of how this rate changed with time.

However, the dependence of the star formation rate on radius could be quite different from an almost linear one, if one considers that the arms play a dominant role. For instance, if we consider that the Lin \& Shu (1964) theory of spiral arms is correct, then the arms should stop abruptly at the Lindblad resonances. The arms, and consequently star formation, should only occur in the region where the pattern speed $\Omega_{p}$ is between $\Omega-\kappa / 2$ and $\Omega+\kappa / 2$, where $\Omega$ is the angular rotation speed of the disk, and $\kappa$ the epicycle frequency. Another prediction, which is discussed below, is that a minimum of star formation rate could appear at corotation, close to the middle between the Lindblad resonances.

There is here an opportunity to test both our understanding of spiral waves, and of the chemical evolution of galactic disks. If the spiral structure of a galaxy is stable, the regions of the disk where there is an enhanced star formation rate will build up a larger metallicity. The study of oxygen abundance gradients is of special interest in this context. First, because oxygen originates almost only in type II supernovae ( Matteucci (2003)), and second, because the type II supernovae originate from massive stars that had not enough time to move out from their birthplaces, and are clearly related to the spiral arms. 


\section{Description of star-formation rate associated with spiral arms}

The fact that spiral arms are the triggering mechanism of star formation is obvious from the images of spiral galaxies, where the OB stars and HII regions are seen in the arms, and from dozens of theoretical papers following Roberts (1969).

In the case of our Galaxy, the recent study of Dias \& Lépine (2005) gives strong support to the fact that all the stars are formed in spiral arms. The authors showed that almost all the open clusters where born in the arms, even if they are presently in an inter-arm region. The result is obtained by integrating backwards the orbits of the clusters for a time equal to their age. That work also gives support to the classical theory of spiral arms of Lin \& Shu (1964), since it shows that the 3 arms observed in the solar neighborhood rotate at the same angular speed, like a rigid body.

One of the simplest recipe, adopted by Mishurov, Lépine \& Acharova (2002), to take into account star formation by the arms, is to consider that the star formation rate (SFR) is proportional to the rate at which the gas feeds the star-forming machine (the arms). This rate is equal to the surface density $\sigma$ of the gas times the relative velocity of the gas $(\Omega)$ with respect to the arms $\left(\Omega_{p}\right)$. In other words,

$$
S F R \propto \sigma\left|\Omega-\Omega_{p}\right|
$$

In our Galaxy, the corotation radius where $\Omega=\Omega_{p}$ is very close to the Sun (eg Dias \& Lépine (2005), and references therein). According to this equation we should have a minimum of star formation rate (and of metallicity) close to the Sun. This equation predicts a gradient of oxygen abundance that rises in the external parts of the Galaxy.

However, the prediction that the star formation rate reaches a value close to zero near the Sun is somewhat puzzling, since such a deep minimum is not observed; the spiral arms do not seem to present any clear interruption in the solar neighborhood. One possible explanation would be that the orbits followed by the gas are not so close to circular ones, for instance the potential of the Galaxy may have larger departure from axis-symmetry than usually considered.

Another possibility, recently investigated by Acharova, Lépine, Mishurov (2005) is that the SFR is proportional to $\sigma$ to some power, but not to $\left|\Omega-\Omega_{p}\right|$. In this last work, it is considered that not the star formation rate, but the term $P z$ (that represents the part of the stellar mass ejected into the ISM as a new synthetized element, in the equations of chemical evolution), depends on $\left|\Omega-\Omega_{p}\right|$.

\section{Main results}

In both approaches of chemical evolution models that take into account the spiral arms as described above, we obtain bimodal oxygen abundance gradients for the Galaxy, with strong gradients at inner radii of the disk, and flat gradient near the Sun, as observed.

\section{References}

Acharova, I.A., Lépine, J.R.D., \& Mishurov, Yu. N. 2005, MNRAS 359, 819

Dias, W.S. \& Lépine 2005, ApJ (in press)

Lin, C.C. \& Shu F.H. 1964, ApJ 155, 721

Matteucci, F. 2003 (in McWilliam A. \& Rauch M., eds, Carnegi Obs. Astrophys. Ser. Vol. 4, Origin and evolution of the elements, Cambrige Univ. Press, p. 1)

Mishurov, Yu. N., Lépine, J.R.D. \& Acharova, I.A. 2002, ApJ 571, L113

Roberts, W.W. 1969, ApJ 158, 123 